\title{
RSS-Ratio for Enhancing Performance of RSS-based Applications
}

\author{
Wei Cheng \\ Department of Computer Science \\ University of Massachusetts Lowell \\ Lowell, MA 01854, USA \\ Email: wcheng@cs.uml.edu
}

\author{
Kefeng Tan, Victor Omwando, Jindan Zhu, Prasant Mohapatra \\ Department of Computer Science \\ University of California, Davis \\ Davis, CA 95616, USA \\ Email: $\{$ kftan, voomwando,jdzhu $\}$ ucdavis.edu, prasant@cs.ucdavis.edu
}

\begin{abstract}
RSS (Received Signal Strength) has been widely utilized in wireless applications. It is, however, susceptible to environmental unknowns from both temporal and spatial domains. As a result, the fluctuation of RSS may degrade performance of RSS based applications. In this work, we propose a novel RSS processing method at the receiver for three antenna based systems. The output of our approach is 'RSS-Ratio', which eliminates the environmental unknowns and thus is a more stable variable compared to RSS itself. To validate the efficacy of the proposed method, we conduct a series of experiments in a range of wireless scenarios, including indoor laptop based measurement, indoor software defined radio - WARP based measurement, and outdoor wireless measurement. In addition, we also give an analysis to the relationship between the location of transmitter and the value of RSS-Ratio, and examine the accuracy of the estimated RSS-Ratio value via both simulations and experiments. All the experimental, analytical, and simulated results demonstrate that RSS-Ratio will be a better replacement for RSS to improve the performance of RSS based applications.
\end{abstract}

\section{INTRODUCTION}

RSS (Received Signal Strength) is an indicator of the power of received radio signal at receiving antennas. It has been widely utilized in wireless systems for applications such as localization [1], distance ranging [2], and security [3]. Broadly, there exists two types of methodologies in utilizing RSS: (i) Find: Derive wireless system parameters based on the given(measured) RSS values, such as the distance from a sender to a receiver; (ii) Record: Analyze the property(distribution) of RSS values under a given wireless system(network) setup (such as the locations of wireless users) for enabling certain applications (such as Sybil attack detections). Under the Find methodology, the applications require stable RSS inputs in both the temporal and spatial domains to ensure the uniqueness of the derived parameters. On the other hand, estimating RSS values accurately is necessary for the Record methods such that the properties of RSS can be clearly derived.

In reality, however, the measured RSS values for Find based applications are susceptible to environmental changes [4], [5], and the models ( such as Equ. (1) ) employed for estimating RSS in Record based applications are too complex to control. For instance, theoretically, the log-normal shadowing model [6] is the widely adopted signal propagation model for analyzing RSS,

$$
P_{r}=P_{0}-10 \alpha \log \left(\frac{d}{d_{0}}\right)+\chi_{\sigma}
$$

where $d$ is the distance between the sender and the receiver, $P_{r}$ is the received power (in $\mathrm{dBm}$ ) at the receiver, $P_{0}$ is the received power (in $\mathrm{dBm}$ ) at the reference distance $d_{0}$, which must be chosen such that it lies in the far-field region, $\alpha$ is the path loss exponent, and the background noise $\chi_{\sigma}$, which is a zero-mean Gaussian distributed random variable (in $\mathrm{dBm}$ ) with standard deviation $\sigma$ (in $\mathrm{dBm}$ ) in case of only shadow fading or slow fading. Note that $\chi_{\sigma}$ may be a random variable with Rayleigh distribution or Ricean distribution when only fast fading caused by multipath propagation is considered [7]. According to [8], the value of the path loss exponent $\alpha$ can be chosen from Table. I based on the type of environment.

\begin{tabular}{|c|c|}
\hline Type of Environment & Pass loss exponent \\
\hline Free Space & 2 \\
\hline Urban area cellular radio & $2.7-3.5$ \\
\hline Shadowed urban cellular radio & $3-5$ \\
\hline In building LOS & $1.6-1.8$ \\
\hline Obstructed in building & $4-6$ \\
\hline Obstructed in factory & $2-3$ \\
\hline TABLE 1. PATH LOSS EXPONENT VALUE
\end{tabular}

Accordingly, at least 7 environmental unknowns and manmade choices can affect the value of RSS $\left(P_{r}\right)$ : (i) sender's transmission power, which will affect $P_{r}$ and $P_{0}$; (ii) measurement error of the power at the reference point (error in $P_{0}$ ); (iii) measurement error of distance at the reference point (error in $d_{0}$ ); (iv) measurement error of the distance at the receiver (error in $d$ ); (v) the chosen value of path loss exponent (error in $\alpha$ ); (vi) the background noise $\left(\chi_{\sigma}\right)$; (vii) system calibrations (including RF radio, antenna, amplifier, and system software).

It is technically impossible to accurately obtain all these environmental unknowns as they may be unavailable, unpredictable, uncontrollable, variable, or too complex. Therefore, the problem of either obtaining stable RSS values or accurately estimating RSS has largely remained open in the literature. As a result, the performance of deriving unknowns based on given RSS values and analyzing RSS property is often unsatisfactory. In other words, the nature of RSS determines that its performance may not be satisfactory in supporting the applications under either type of methodologies.

Regardless of the problems we mentioned, RSS is still very attractive for wireless applications in reality, because it is inherent (does not depend on any additional hardware) and always available (sometimes it is the only available information). It is, therefore, worth further investigating RSS to improve its stability and predicability. To this end, we conduct 
an updated RSS research, which is based on two observations: (i) with the prevalence of multiple antennas, multiple RSS values are available from today's wireless devices (such as the IEEE 802.11n enabled devices); (ii) combining multiple RSS values may yield a desired result. In particular, we propose a novel RSS processing method based on the readings from three antennas. The output value becomes stable and predictable by reducing the number of environmental unknowns. Note that the minimum required number of antennas to eliminate most of the environmental unknowns is three, according to the analytical and experimental results of this research, and that two antennas are not sufficient to yield stable RSS related outputs [3]. The contributions of this paper are summarized at the followings:

- We propose a novel three-antenna based RSS processing method, named RSS-Ratio, whose output is a ratio of RSS values. It eliminates most of the environmental unknowns that affect the stability of the output values.

- We conduct a wide range of experiments to evaluate the stability of RSS-Ratio via varying the environment (indoor and outdoor), the platform (Laptops, WARP, Soekris), the location of receiver, the transmission power of transmitter, and the wireless band $(2.4 \mathrm{GHz}$ and $5 \mathrm{GHz}$ ).

- We empirically verify that RSS-Ratio is more stable and concentrated than RSS. Therefore, it is a more desirable replacement for RSS to support the applications under the Find methodology.

- We demonstrate the property of RSS-Ratio through numerical analysis and simulations. The presented results can be used to estimate the value of RSSRatio given the system setup, and the estimation of RSS-Ratio is quite accurate. As a result, RSS-Ratio is a better choice than RSS to enable the applications under the Record methodology.

The rest of the paper is organized as follows. The proposed three-antenna based RSS processing method and the definition of RSS-Ratio are introduced in Section II. To justify the advantage of applying RSS-Ratio to Find based applications, we compare the measured RSS-Ratio with the measured RSS from a laptop based indoor testbed, a WARP based indoor testbed, and our outdoor wireless network testbed (QuRiNet) in Section III, Section IV, and Section V, respectively. Section VI analyzes the property of RSS-Ratio and points out the potential of employing it to enable the applications under the Record methodology. We briefly review the related work in Section VII, followed by the conclusion in Section VIII.

\section{THREE-ANTENNA BASED RSS PROCESSING}

In order to eliminate the environmental unknowns, we consider utilizing three RSS readings from three closely colocated antennas, which are readily available at many IEEE $802.11 \mathrm{n}$ enabled devices. We assume that all the environmental unknowns are identical to the three antennas at any instant of time except for their distances to the signal sender, denoted by $d_{1}, d_{2}$, and $d_{3}$, respectively.

Let $P_{r}^{1}, P_{r}^{2}$ and $P_{r}^{3}$ denote the RSS readings of antenna 1 , antenna 2 and antenna 3 respectively. According to Equ. (1), we have

$$
P_{r}^{1}-P_{r}^{2}=-10 \alpha \log \left(\frac{d_{1}}{d_{2}}\right)
$$

where the environmental unknowns $P_{0}, d_{0}$, and $\chi_{\sigma}$ in Equ. (1) have been eliminated. Furthermore, through jointly processing the RSS readings of all the three antennas, we have

$$
\frac{P_{r}^{1}-P_{r}^{2}}{P_{r}^{1}-P_{r}^{3}}=\frac{\log \left(\frac{d_{1}}{d_{2}}\right)}{\log \left(\frac{d_{1}}{d_{3}}\right)}
$$

where the path loss exponent $\alpha$ has also been eliminated. Note that all the linear and modulus unknowns in Equ. (1) can be eliminated through the above two processing steps. Then, we have the formal definition of RSS-Ratio at the following:

Definition 1: (RSS-Ratio), denoted by $\tau$, is the output value of the three-antenna based RSS processing. Formally,

$$
\tau=\frac{P_{r}^{1}-P_{r}^{2}}{P_{r}^{1}-P_{r}^{3}}=\frac{\log \left(\frac{d_{1}}{d_{2}}\right)}{\log \left(\frac{d_{1}}{d_{3}}\right)}
$$

One can see that the value of RSS-Ratio only depends on the distances from the sender to the three receiving antennas. Intuitively, a RSS-Ratio should be more stable than a RSS as there exists only one environmental unknown $d$, which is not a time-varying variable if the device is stationary. Thus, theoretically, RSS-Ratio should be a desirable RSS replacement for improving the performance of the applications under the Find methodology. In Sec. III-V, we demonstrate the superior stability of RSS-Ratio via experiments. In addition, the expected RSS-Ratio can be uniquely calculated based on Equ. (4), provided the location of the senders and the three receiving antennas are known. Therefore, it can also replace RSS for the applications under the Record methodology. In Sec. VI, we study the property of the RSS-Ratio value distribution via both analysis and simulations. Note that, theoretically, more antennas (such as $4,5, \ldots$ ) at the receiver cannot further eliminate the distance unknowns from Equ. (4) as each antenna also brings in its own distance unknown.

\section{INDOOR LAPTOP BASED EXPERIMENTS}

We first examine the stability of RSS-Ratio through the indoor laptop based experiments as shown in Fig. 1(a), where the black dot represents the location of the receiver and the black rectangle indicates the location of the sender. All the experiments are conducted on a $2.4 \mathrm{GHz}$ frequency band on the second floor of a two-story office building, whose room is furnished with desks, chairs, computers, and cabinets.

\section{A. Experiments Setup}

In the experiments, the receiver is a Dell E5400 laptop equipped with three antennas at the top left, at the top right, and at the top center of the LCD screen, respectively. The sender is also a Dell E5400 laptop that sends ping packets with $10 \mathrm{~ms}$ interval to the receiver via a single antenna (two of its three antennas have been disabled). Similar to [3], the receiver is running on a modified Fedora Linux kernel version 2.6.29-rc5-wl. The wireless device driver, the kernel-to-user space communication library (radiotap), and Tcpdump are all modified to obtain the automatic gain control (AGC) and RSS reading of each frame received by the three antennas, 


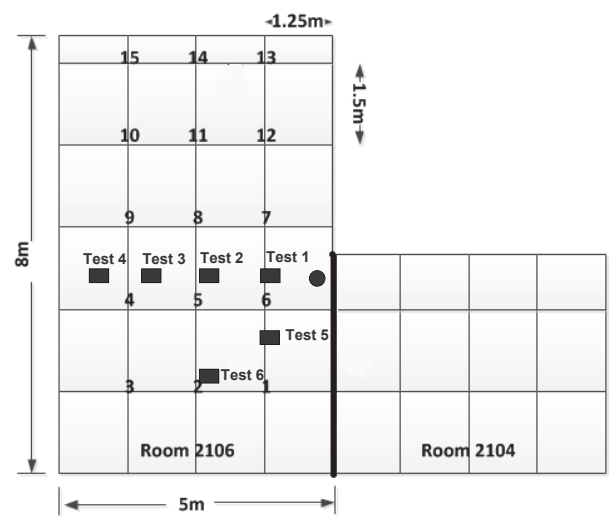

(a) Laptop Test Map

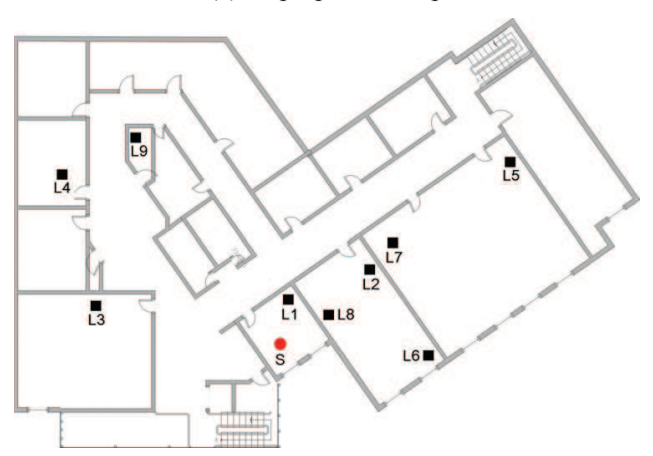

(b) WARP Test Map
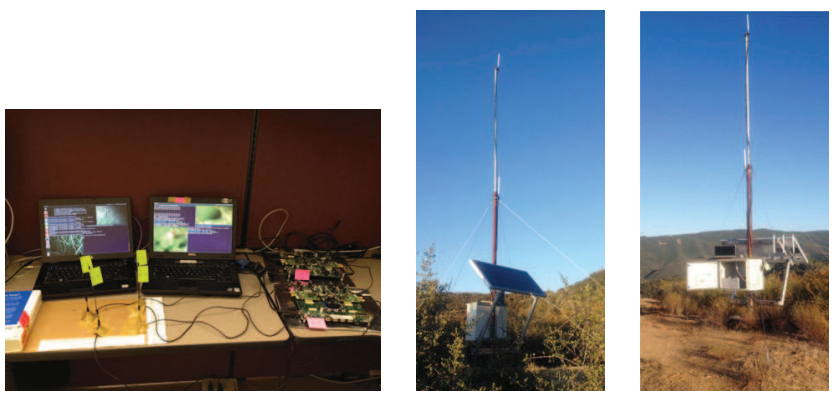

(c) WARP Setup

(d) Outdoor Site- (e) Outdoor SiteA-TX B-RX

Fig. 1. Experiment setup

respectively. The RSS value can be converted to $\mathrm{dBm}$ via: $R S S(d B m)=R S S$ (reading) $-A G C-O F F S E T$, where OFFSET is 44 , a constant set by the system's Wi-Fi module, and AGC is identical to the three antennas but variable to each frame. In addition, both the sender and the receiver are configured in ad-hoc mode, and placed on the same type of desks stationarily. As shown in Fig. 1(a), the locations of the sender are aligned along two angles to the receiver with an increasing distance from 1 meter to 4 meters at a step of 1 meter. For each location, the sender continuously generates ICMP traffic at a rate of approximately $12 \mathrm{Mb} / \mathrm{s}$ for 5 minutes. The modified Tcpdump at receiver captures the traffic and reports all three RSS readings of each frame from individual antenna.

\section{B. $2.4 G H z$ Frequency Tests}

Fig. 2 compares RSS and RSS-Ratio values over a $2.4 \mathrm{GHz}$ band. It can be observed that the distribution of the RSSRatio is generally more concentrated than the distribution of the measured RSS readings except in test 4 . We conjecture that the exception can be attributed to three reasons. (i) The shape of the receiver's antennas cannot be considered as single points in the indoor environment because of the impact of multipath, and the relative directions from the sender's antenna to the receiver's antennas. (ii) The stability of RSS-Ratio is also affected by the system configurations, such as the range of RSS reading and the automatic rate adaptation, which causes the RSS to depart from the model in Equ. (1). The RSS reading reported by the wireless driver (Intel iwlwifi) is an integer in a fixed range. This range is usually much smaller than the dynamic range of the actual received signal strength [3]. As a result, the reported RSS value may clipped at the upper (or lower) bound if the transmission power is too high (or too low) or the distance is too small (or too large). The automatic rate adaptation triggers changes to the modulation of preamble at the physical layer, which can cause a large variation in reported RSS values. For example, the OFDM modulation scheme is utilized by $802.11 \mathrm{~g}$ when the data rate is $54 \mathrm{Mbps}$, while the CCK modulation scheme will be activated if the date rate is decreased to $11 \mathrm{Mbps}$ or lower.(iii) the interference can also affect the RSS readings as $2.4 \mathrm{GHz}$ band is typically very crowded in office buildings.

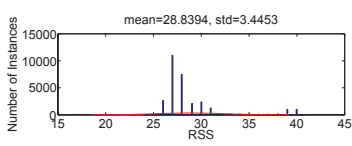

(a) Test 1-RSS

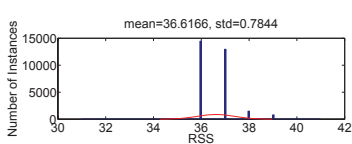

(c) Test 2-RSS

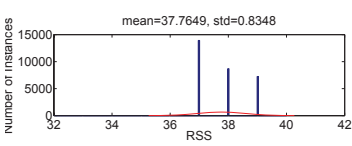

(e) Test 3-RSS

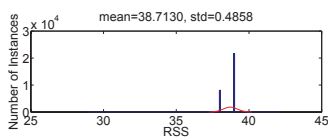

(g) Test 4-RSS

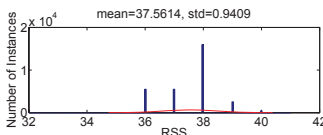

(i) Test 5-RSS

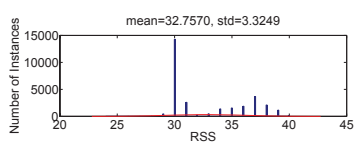

(k) Test 6-RSS

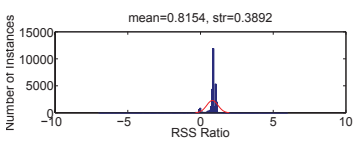

(b) Test 1-RSS Ratio

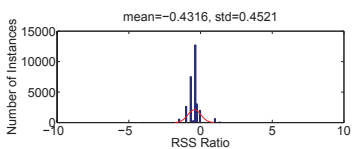

(d) Test 2-RSS Ratio

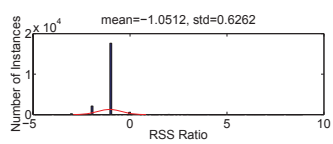

(f) Test 3-RSS Ratio

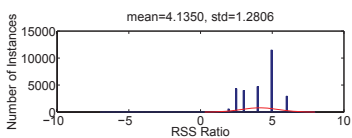

(h) Test 4-RSS Ratio

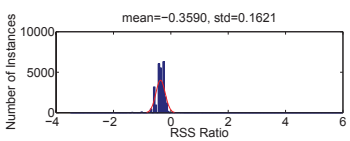

(j) Test 5-RSS Ratio

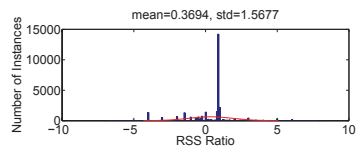

(1) Test 6-RSS Ratio
Fig. 2. The measured RSS and RSS-Ratio on $2.4 \mathrm{GHz}$ band in the laptop based tests. 


\section{INDOOR WARP BASED EXPERIMENTS}

Although the indoor laptop results verified our approach, we realize two important insufficiency of the experiments. First, the relative position of three receiving antennas at laptop can not be adjusted even if the relative distance between the sender and the receiver is changed. Second, most of the Wi-Fi cards on laptops (including ours) only support operations over $2 \mathrm{GHz}$ band. To exclude the potential dependency on relative position of receiving antennas and on operational band, we switch to a new platform - Wireless Open Access Research Platform (WARP) [9]. WARP is a software defined radio (SDR) based platform, and allows a more flexible control to overcome the insufficiency we mentioned.

As shown in Fig. 1(b), two WARP boards with four antennas (Fig. 1(c)) are placed at the red dot as receivers. The signal sender, a Dell E5400 laptop, is placed at the black squares. Note that we only use three out of the four antennas. In the WARP based tests, the stability of RSS-Ratio is examined at varied central frequency, transmission power, and receiving antenna locations.

\section{A. Experiment Setup}

WARP can support two external antennas, whose positions can be freely adjusted. It has an easy access to RSS value from each antenna. One WARP can provide two RSS readings for a received frame as its real-time OFDM reference design v16.1 only supports $2 \times 2$ MIMO configurations. Therefore, in order to acquire three RSS readings for the same frame, we need to use two WARP boards as the receiver.

As we only have two WARP available, we have to use the laptop as the signal sender. In order to detect the start of a frame from the sender, WARP continuously samples the incoming signals. If the power of received signal is higher than the energy threshold that is preset above the noise floor, WARP self-correlates the incoming samples with itself and cross-correlates the samples with a local sequence that is in the same pattern as the preamble of the received frame. Note that the preambles of WARP and IEEE $802.11 \mathrm{a} / \mathrm{g}$ card have the same pattern as their PHY layers both use OFDM with 52 subcarriers. Then, the frame is identified and its RSS is calculated and outputted to a register if both of the correlations have passed their corresponding thresholds.

Note that WARP's operational bandwidth is $10 \mathrm{MHz}$ and not $20 \mathrm{MHz}$ (the one used in IEEE $802.11 \mathrm{a} / \mathrm{g}$ ). Consequently, it is not compatible with IEEE $802.11 \mathrm{a} / \mathrm{g}$ wireless interface cards. In other words, the receiver (WARP) cannot decode the frame from the sender (laptop) due to their PHY layer difference. For our purpose, we only need to acquire the RSS readings of an incoming frame, which is feasible even if WARP cannot decode the whole frame. However, it brings an additional problem when combining the RSS readings from the two WARP boards as the corresponding RSSs for the same frame cannot be linked together due to the lack of frame ID. No matter how carefully we adjust the thresholds on the two boards, there always exist differences in detection sensitivity as well as in the respective channel condition. As a result, we cannot guarantee that the two boards can always detect the same set of frames. Therefore, a particular problem is how to synchronize the two WARP boards such that the three RSS readings (from two WARP boards) for a same frame can be identified.

For the synchronization, the challenge is that no sequence number or other information can be used to differentiate the frames because the WARP can neither decode any contents of frames nor provide any timestamp for each frame due to the lack of operating system. One possible solution is to connect two boards and synchronize the hardwares. This approach, however, takes non- trivial modifications to the PHY layer. We hereby take a software-based approach. In our synchronization method, each WARP board will output the RSS to its connected laptop via serial port once a frame has been detected. The laptop record the RSS reading in a log file. We connect the two laptops through Ethernet, and monitor the updates on the two files using inotifywait. We consider both boards have successfully detected the same frame if two new RSS readings have been written into their corresponding files simultaneously. Otherwise, the new RSS reading will be removed from the log files. Particularly, we set the interval of frame transmission as $50 \mathrm{~ms}$ at the sender, so that the interval of two continuous RSS writings at the same log file is long enough from being considered as simultaneous. Note that, in the following tests, the reported RSS values are the original WARP RSS readings, which can be converted to the corresponding values in $\mathrm{dBm}$ by $R S S(d B m)=0.072 \times R S S($ reading $)-103$.

\section{B. $5 \mathrm{GHz}$ Frequency Tests}

Fig. 3 reports the measured RSS reading and the RSS-Ratio on a $5 \mathrm{GHz}$ band from the locations L1-L7. The $5 \mathrm{GHz}$ band is quite clean in our lab. The results are consistent with the general observations we obtained from the laptop based tests, which are conducted on $2.4 \mathrm{GHz}$ band. Thus, the distribution of the measured RSS-Ratio is generally more concentrated than the distribution of the measured RSS over the two most popular ISM bands $(2.4 \mathrm{G}$ and $5 \mathrm{G})$ whatever the channels are saturated or not.

\section{Transmission Power Tests}

Since RSS is a signal power indicator, the change of transmission power should directly affect the RSS readings at the receiver. According to Equ. (1), the receiver is unable to derive the system parameters (Find methodology) and to utilize RSS (Record methodology) if the sender's transmission power is unknown. On the contrary, theoretically, the applications based on RSS-Ratio can still succeed even without the awareness of the sender's transmission power as $P_{0}$ has been eliminated from Equ. (4).

The wireless card utilized by the sender (Dell E5400 laptop) has 5 available transmission power levels (TX6, TX8, TX10, TX12, and TX14). We adjust its transmission power via utilizing madwifi [10]. The measured results over transmission power at location L2, L3 and L4 are reported in Fig. 4, Fig. 5, and Fig. 6, respectively.

One can see that the distribution of the measured RSSRatio is still more concentrated than the distribution of the measured RSS under the variation of transmission power. Fig. 7 summarizes the variations of RSS and RSS-Ratio over transmission power. The gradients of RSS-Ratio curves in Fig. 7(b) are smaller than the corresponding ones of RSS in 


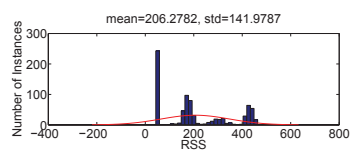

(a) L1-RSS

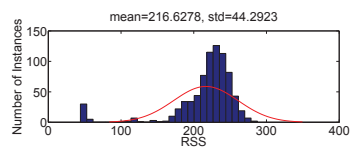

(c) L2-RSS

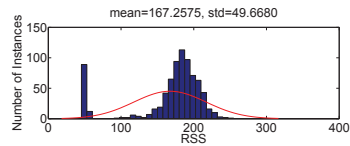

(e) L3-RSS

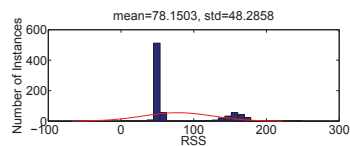

(g) L4-RSS

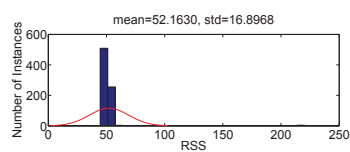

(i) L5-RSS

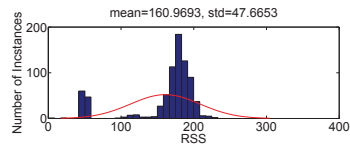

(k) L6-RSS

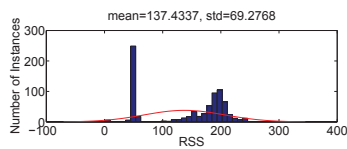

(m) L7-RSS (b) L1-RSS Ratio

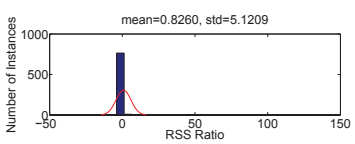

(d) L2-RSS Ratio
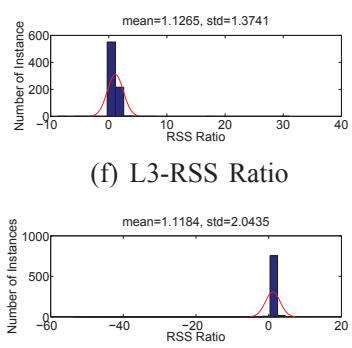

(h) L4-RSS Ratio

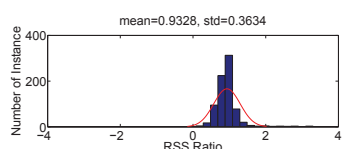

(j) L5-RSS Ratio

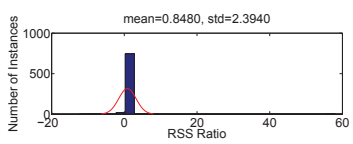

(1) L6-RSS Ratio

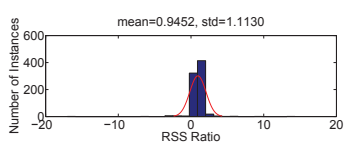

(n) L7-RSS Ratio

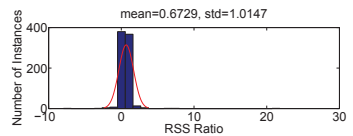

(f) L3-RSS Ratio

Fig. 3. The measured RSS and RSS-Ratio on $5 \mathrm{GHz}$ band in the WARP based tests.

Fig. 7(a). It means that the variation of RSS-Ratio is relatively smaller than the variation of RSS. The exceptions happen at L2-TX14, L3-TX14, and L3-TX10. They look like normal randomness, but we will further investigate the possible reasons for the exceptions in our future work via more experiments with different types of senders.

\section{Antenna Location Tests}

The change of the receiving antenna locations could completely change the environmental unknowns such as the path loss exponent, and change the values of RSS and RSS-Ratio accordingly. It is therefore necessary to examine the stability of RSS-Ratio via varying the receiving antenna locations. Note that the receiving antenna locations in Sec. IV-B are different from the ones in Sec. IV-C. In order to further justify the stability of RSS-Ratio, we move the receiver to two additional locations. The measured results of the sender locations L1, L3, L6, L8 and L9 at the two additional receiving locations are reported in Fig. 8 and Fig. 9, respectively. It is clear that the distribution of the measured RSS-Ratio is always more

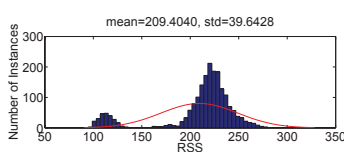

(a) L2-TX6 RSS

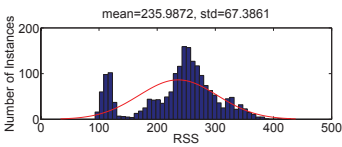

(c) L2-TX8 RSS
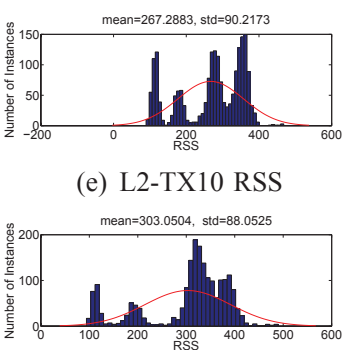

(g) L2-TX12 RSS

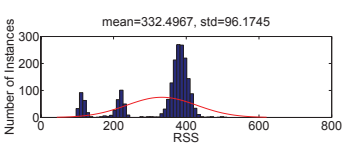

(i) L2-TX14 RSS (e) L2-TX10 RSS

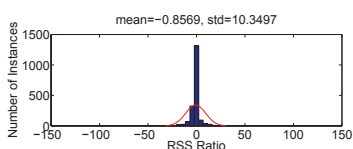

(b) L2-TX6 RSS Ratio

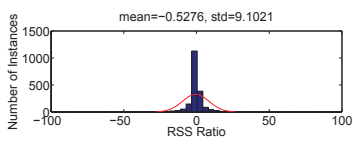

(d) L2-TX8 RSS Ratio

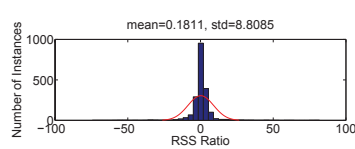

(f) L2-TX10 RSS Ratio

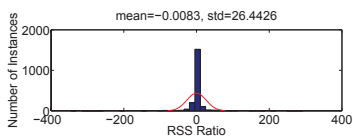

(h) L2-TX12 RSS Ratio

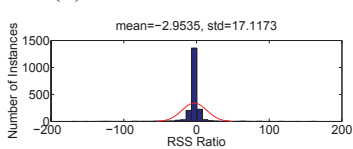

(j) L2-TX14 RSS Ratio
Fig. 4. The measured RSS and RSS-Ratio over transmission powers in the WARP based tests at location L2.

concentrated than the distribution of the measured RSS though the particular values of RSS and RSS-Ratio may vary.

In summary, based on all the results presented in Sec. III and IV, we can say that the proposed RSS-Ratio is more stable than RSS over different system setups (such as sender location, receiver location, transmission power, frequency, interference, and platform) in indoor environments.

\section{OUtDOOR QuRiNet EXPERIMENTS}

All previous experiments are conducted indoor, we therefore present outdoor experiments in this section to show the applicability of RSS-Ratio in an outdoor environment. We leverage the Quail Ridge Wireless Multihop Testbed for our outdoor experiments(QuRiNet) [11]. Besides the obvious difference in wireless environment between QuRiNet and the office settings that significantly affect the propagation of RF signals over the air, QuRiNet has an unique property that we have not explored in previous experiments. Because QuRiNet is located in a remote nature reserve area, the environment is free of external RF interference. In another word, the interference to the testing link is under the control if configured properly. As interference widely exist in real networks, QuRiNet provides an unique opportunity for us to validate RSS-Ratio with controlled interference.

We first explain the configuration of QuRiNet. QuRiNet is an outdoor, solar-powered wireless testbed deployed in the Quail Ridge Reserve at Lake Berryessa, California. It comprises of forty one sites spread over and area of approximately 2000 acres. Each site has a Soekris net4826 embedded computer running the OpenWRT OS from the latest trunk sources 


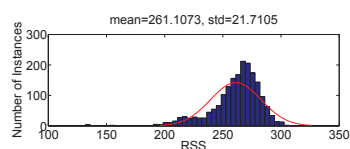

(a) L3-TX6 RSS

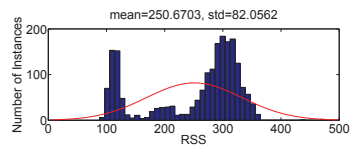

(c) L3-TX8 RSS

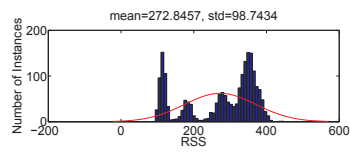

(e) L3-TX10 RSS

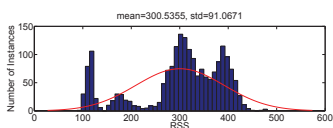

(g) L3-TX12 RSS

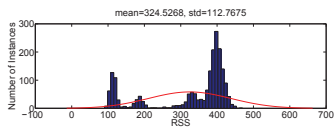

(i) L3-TX14 RSS

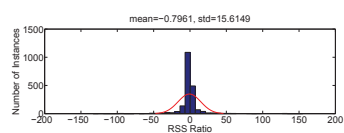

(b) L3-TX6 RSS Ratio

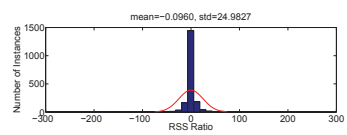

(d) L3-TX8 RSS Ratio

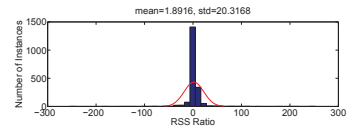

(f) L3-TX10 RSS Ratio

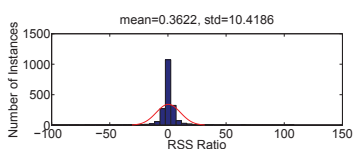

(h) L3-TX12 RSS Ratio

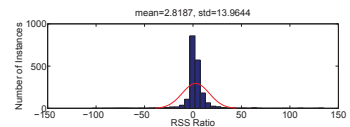

(j) L3-TX14 RSS Ratio
Fig. 5. The measured RSS and RSS-Ratio over transmission power in the WARP based tests at location L3.

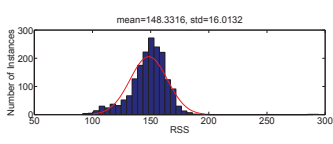

(a) L4-TX6 RSS

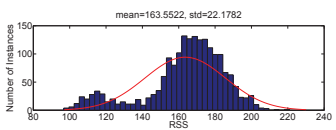

(c) L4-TX8 RSS

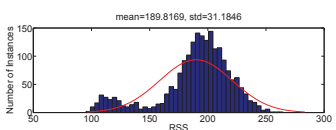

(e) L4-TX10 RSS

(g) L4-TX12 RSS

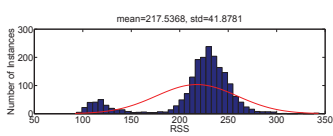

(i) L4-TX14 RSS

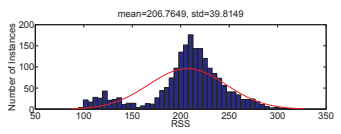

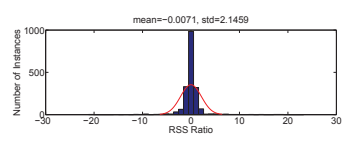

(b) L4-TX6 RSS Ratio

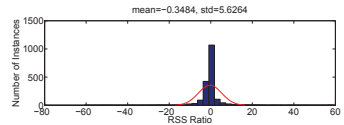

(d) L4-TX8 RSS Ratio

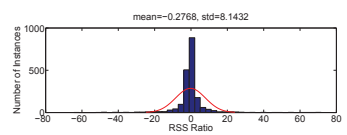

(f) L4-TX10 RSS Ratio

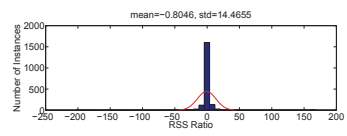

(h) L4-TX12 RSS Ratio

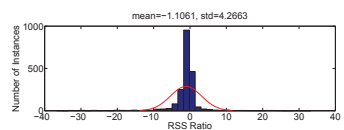

(j) L4-TX14 RSS Ratio
Fig. 6. The measured RSS and RSS-Ratio over transmission power in the WARP based tests at location L4.

("Attitude Adjustment") [12] with Linux kernel version 3.3.8. The routers are linked using the ad-hoc 802.11 IBSS mode, with the Open Link State Routing protocol (OLSR) providing routing at the IP layer [13]. Since the area is uninhabited, it gives us an excellent chance to deploy large-scale experiments

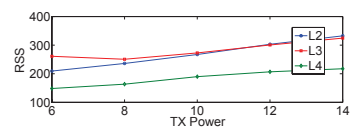

(a) RSS

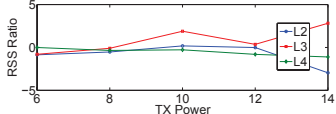

(b) RSS Ratio
Fig. 7. The TX power impacts on RSS and RSS-Ratio in the WARP based tests.

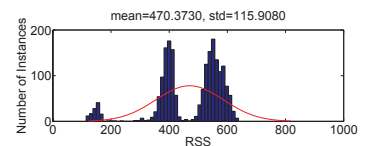

(a) L1 RSS

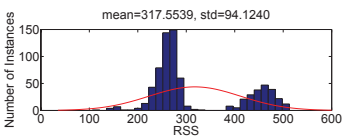

(c) L3 RSS

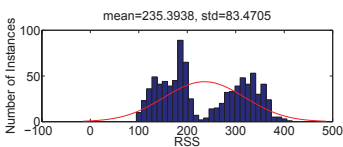

(e) L6 RSS

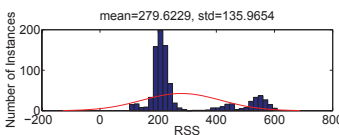

(g) L8 RSS

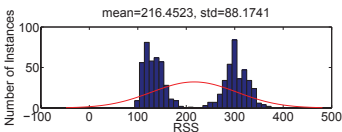

(i) L9 RSS

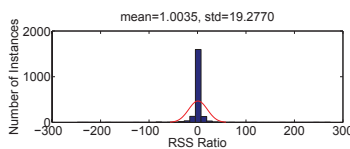

(b) L1 RSS Ratio

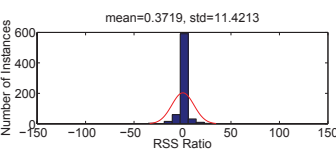

(d) L3 RSS Ratio

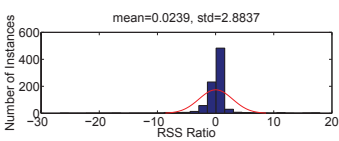

(f) L6 RSS Ratio

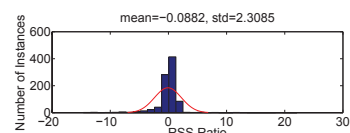

(h) L8 RSS Ratio

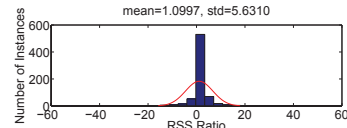

(j) L9 RSS Ratio
Fig. 8. The measured RSS and RSS-Ratio at additional location-(1) in the WARP based tests.

in an area that is largely free of electromagnetic interference and other sources of perturbation.

\section{A. Experiment Setup}

For our purpose, we deliberately select two different sites which are 371 meters apart with minor foliage obstruction between them. We designate site A (Fig. 1(d)) as the sender with one 2x2 Microtik R52n-M MIMO Mini-PCI form-factor wireless card which uses the Artheros AR9220chipset and the ath9k open source wireless driver. At the receiving site B (Fig. 1(e)), we set up one router with dual wireless cards. All three wireless interfaces at both sites have an omni- directional antenna with $7.4 \mathrm{dBi}$ antenna gain. We attach two antennas on the first card and one on the other. At site B, the three antennas are set in a line six inches $(15 \mathrm{~cm})$ apart such that there is a 12 inch $(30 \mathrm{~cm})$ gap between the first antenna and the third one. The antenna at site $\mathrm{A}$, mounted on a 3.1 meter mast, has an altitude of 392 meters, while the three antennas at site B have an altitude of 399 meters.

The experiments are conducted at the frequency of 2.412 $\mathrm{GHz}$, and there exists co-channel interference from five other sites in the environment; the next closest site is 436 meters from the receiving site $\mathrm{B}$. All the other sites have a fixed 


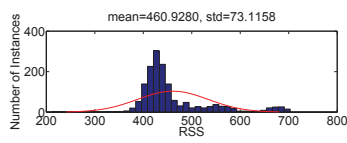

(a) L1 RSS

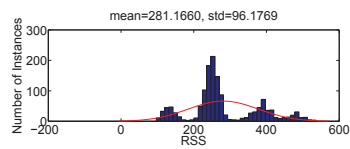

(c) L3 RSS

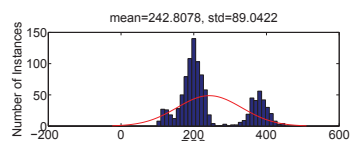

(e) L6 RSS

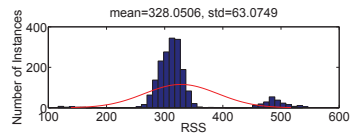

(g) L8 RSS

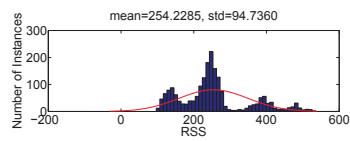

(i) L9 RSS

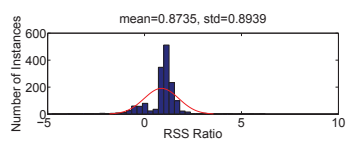

(b) L1 RSS Ratio

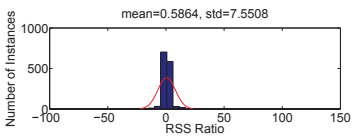

(d) L3 RSS Ratio

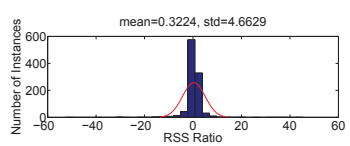

(f) L6 RSS Ratio

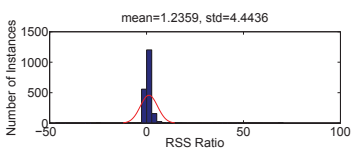

(h) L8 RSS Ratio

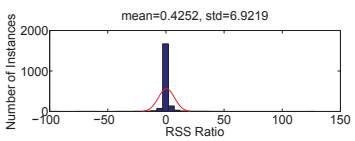

(j) L9 RSS Ratio
Fig. 9. The measured RSS and RSS-Ratio at the additional location-(2) in the WARP based tests.

transmission power of $16 \mathrm{dBm}$, with $7 \mathrm{dBi}$ omni-directional antennas. Further, at site $\mathrm{B}$, we collect RSS readings of transmissions from site $\mathrm{A}$, repeating the scenario with the TX power at $6,810,12,14$ and $16 \mathrm{dBm}$. We use the $i w$ user-space command at A to dynamically set fixed transmission powers.

Frame transmission is achieved by the periodic beaconing done as part of the IBSS management. At site B, we make modifications to the ath9k driver to print kernel debug messages for the RSS values of received frames at each antenna; by default, the driver aggregates the RSS value for the antennas at each card. Specifically, we add printk() statements in the ath9k_process_rssi function to print the values of the $r s \_r s s i \_c t l[X]$ data members of the ath_rx_status structure. Since the frames that have been received from the single TX antenna have the same frame sequence number, we are able to correlate the RSS readings that have been received at each antenna. The readings can be converted to their respective $\mathrm{dBm}$ values by $R S S(d B m)=R S S($ reading $)-95$.

\section{B. Outdoor Tests}

Theoretically, the outdoor RSS and RSS-Ratio should more strictly follow the signal propagation model in Equ. (1). As a result, we are expecting to observe more concentrated RSSRatio from the outdoor tests. The measured RSS and RSSRatio from QuRiNet are reported in Fig. 10. Intuitively, the distribution of the outdoor RSS-Ratio is also more concentrated than the one of RSS. Fig. 10(m) summarizes the TX power's impacts on the values of RSS and RSS-Ratio in the outdoor environment. One can see that both curves can be considered as straight lines. Similar to the observations obtained from Fig. 7, where the TX power indoor impacts were reported, the gradient of RSS-Ratio line is much smaller than the one of RSS line. As a result, RSS-Ratio is also more stable than RSS in the outdoor environment. Moreover, by comparing the results reported in Fig. 10(m) and Fig.7(b), where the indoor RSS-Ratio variation has been reported, the maximum and the average differences between any two outdoor mean RSS-Ratio values are much smaller than the corresponding ones obtained from the indoor tests. It means that the outdoor RSS-Ratio can be recognized as more stable than the indoor RSS-Ratio, which is consistent with the observation obtained from the theoretical view.

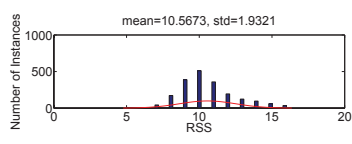

(a) Outdoor-TX6 RSS

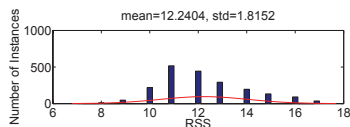

(c) Outdoor-TX8 RSS

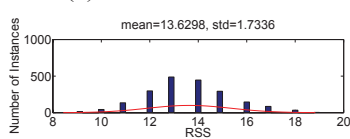

(e) Outdoor-TX10 RSS

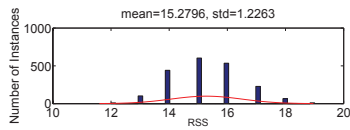

(g) Outdoor-TX12 RSS

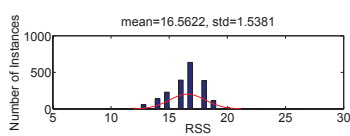

(i) Outdoor-TX14 RSS

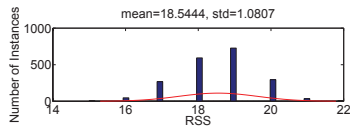

(k) Outdoor-TX16 RSS

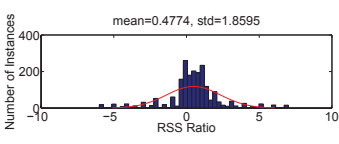

(b) Outdoor-TX6 RSS Ratio

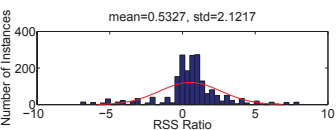

(d) Outdoor-TX8 RSS Ratio

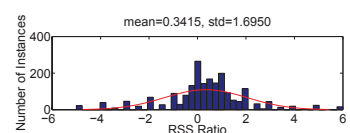

(f) Outdoor-TX10 RSS Ratio

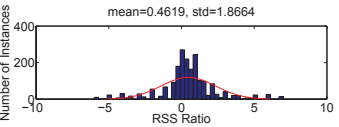

(h) Outdoor-TX12 RSS Ratio

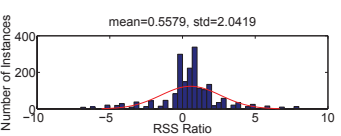

(j) Outdoor-TX14 RSS Ratio

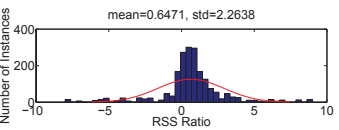

(1) Outdoor-TX16 RSS Ratio

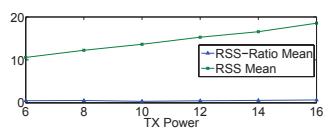

(m) Outdoor TX Impact

Fig. 10. The measured RSS and RSS-Ratio over transmission power in QuRiNet based tests.

\section{RSS-RATIO ANALYSIS}

The experimental results from Sec. III-V have justified that RSS-Ratio is more desirable than RSS to support the applications under the Find methodology. In this section, we demonstrate that RSS-Ratio is also a better choice for the applications under the Record methodology.

\section{A. RSS-Ratio Property Analysis}

Given the system setups such as the locations of the three receiving antennas, we first analyze the relationship between the locations of senders and the values of RSS-Ratio. Note that 
it is not necessary to know the antenna orientation. Without loss of generality, we assume that $(x, y)$ is the coordinates of a sender, and that $\left(x_{1}^{\prime}, y_{1}^{\prime}\right),\left(x_{2}^{\prime}, y_{2}^{\prime}\right)$ and $\left(x_{3}^{\prime}, y_{3}^{\prime}\right)$ are the coordinates of the three receiving antennas, respectively. According to Equ. (4), we have the following function:

Definition 2: 2D Three-Antenna-Ratio function $f_{\tau}^{2 D}$ :

$$
f_{\tau}^{2 D}\left(x, y, x_{1}^{\prime}, x_{2}^{\prime}, x_{3}^{\prime}, y_{1}^{\prime}, y_{2}^{\prime}, y_{3}^{\prime}\right): \frac{\log \frac{\left(x-x_{1}^{\prime}\right)^{2}+\left(y-y_{1}^{\prime}\right)^{2}}{\left(x-x_{2}^{\prime}\right)^{2}+\left(y-y_{2}^{\prime}\right)^{2}}}{\log \frac{\left(x-x_{1}^{\prime}\right)^{2}+\left(y-y_{1}^{\prime}\right)^{2}}{\left(x-x_{3}^{\prime}\right)^{2}+\left(y-y_{3}^{\prime}\right)^{2}}}=\tau
$$

Assuming the locations of the three antennas are given, the relationship between the value of RSS-Ratio and the sender's position can be derived via solving $(x, y)$ from $f_{\tau}^{2 D}$ for each $\tau$ by mathematical tools such as Matlab and Mathematica. Fig. 11(a) and Fig. 11(b) report the relationships when the three antennas are deployed linearly $($ at $(0,0),(1,0)$ and $(2$, $0)$ ) and non-linearly $($ at $(0,0),(1,0)$ and $(0,1))$, respectively. In the figures, each number beside a line is a value of RSS-Ratio $(\tau)$, and the points on the line are the possible solutions of $(x, y)$ for this $\tau$. In other words, each line contains all the possible locations of the senders who may yield the given $\tau$ at the given receiver. Therefore, given a value of RSSRatio, the locations of senders can be clearly estimated by the receiver. On the other hand, the value of RSS-Ratio can also be derived according to Equ. (4) by the receiver given the sender's location. It means that the analysis on the property of RSS-Ratio is almost deterministic.

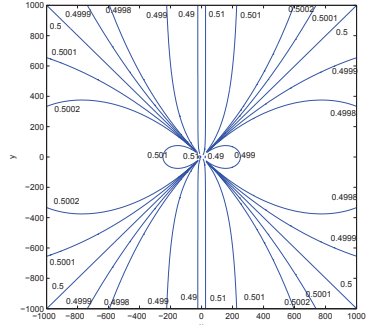

(a) Linear deployment

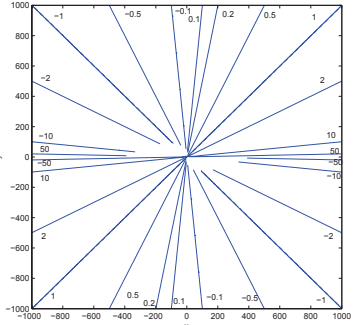

(b) Non-linear deployment
Fig. 11. The relationship between the locations of senders and RSS-Ratio in 2D: (a) The three antennas are deployed linearly at $(0,0),(1,0)$ and $(2,0)$. (b) The three antennas are deployed non-linearly at $(0,0),(1,0)$ and $(0,1)$.

In addition, it is interesting to observe that almost all the sender's possible locations appear on a straight line for any given $\tau$ in Fig. 11(b). It is a beneficial for the receivers that may not have enough computing capability to solve $f_{\tau}^{2 D}$ instantly as a straight line can be constructed by only sampling two reference points. Note that for the area that are close to the receiver, the solutions of $(x, y)$ may not exist or not appear on a straight line.

\section{B. Estimated RSS-Ratio Accuracy Analysis}

Considering the error of localizing a sender is available, we study its impact on the accuracy of the estimated RSS-Ratio in this subsection. We define the square ratio error, denoted by $E^{2}$, as $E^{2}=\left(\tau_{r}-\tau_{e}\right)^{2}$, where $\tau_{r}$ is the accurate value of RSSRatio and $\tau_{e}$ is the estimated value of RSS-Ratio. Assuming the sender's localization error is a 2-dimensional zero-mean
Gaussian distributed random variable, we first analyze the accuracy of estimated RSS-Ratio via simulations.

In the simulations, the three antennas are deployed at at $(0$, $0),(1,0)$ and $(0,1)$, respectively. We randomly generate 100 locations for the sender. For each location, we calculate its $\tau_{r}$ according to Equ. (4), and generate 100 location error instances that follow the 2-demential zero-mean Gaussian distribution for each standard deviation $\sigma$, which varies from 1 to 10 . Then, for each location error instance, we add it to the corresponding sender location and calculate $\tau_{e}$. Fig. 12(a) reports the square ratio error in the simulations. Note that each point in the figure is the average of 100 instances. It can be observed that the square ratio error is relatively low in most of the cases. The high square ratio error is probably caused by a few instances, which yield very large square ratio error, as the reported result is an average and most of the results are very small.

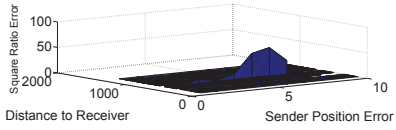

(a) Impact of Location Error

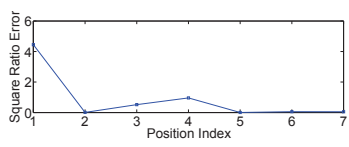

(b) Error in WARP Test
Fig. 12. (a)Square ratio error vs. sender's distance to receiver and sender's location error: The three antennas are deployed at $(0,0),(1,0)$ and $(0,1)$. (b) Square ratio error in the WARP based test

Furthermore, we evaluate the accuracy of estimated RSSRatio based on the experiments in Fig. 3. In those experiments, the three antennas are deployed at $(0 \mathrm{~cm}, 0 \mathrm{~cm}),(-11 \mathrm{~cm}$, $11 \mathrm{~cm})$, and $(0 \mathrm{~cm},-11 \mathrm{~cm})$, respectively. The locations of L1-L7 are manually measured. The localization error is about $35 \mathrm{~cm}$. The measured values of RSS-Ratio reported in Fig. 3 and the values of RSS-Ratio calculated based on the measured sender locations are utilized as $\tau_{r}$ and $\tau_{e}$, respectively. Fig. 12(b) reports the square ratio error in the experiments. One can see that the result is consistent with the one in Fig. 12(a). The square ratio error is relatively low at most of the locations. In summary, RSS-Ratio is more predictable than RSS. It is a better choice for the applications under the Record methodology as its property is clear.

\section{RELATED WORKS}

Received Signal Strength is an useful parameter widely existed in many wireless devices, and can be used to derive the environmental information such as distance and location.

It has been utilized in the wireless applications for either obtaining the information or representing the information. In the literature, the RSS based applications can be classified into two categories by their methodologies of utilizing RSS. (i) The applications that target on acquiring environmental information via analyzing the measured RSS based on the signal propagation model. (ii) The applications that employ the measured or estimated RSS as an environmental information signature to enable related functions.

Under the first category, RSS can be used to estimate the distance for multi-lateration base localization [2]. The authors analyze various environmental factors that can affect the accuracy of distance ranging, and quantify their effects on the resulting localization error. In addition, Dil et al. analyze and compare the best available RSS based localization 
algorithms [14]. Its indoor experimental results demonstrate that RSS ranging based localization algorithms generally outperform the signature based and proximity based localization algorithms when the calibration of RSS measurements is not sufficient.

Under the second category, RSS signature map based localization represents one of the popular applications [1]. In order to obtain a stable RSS related value (signature), a relative long training phase is required to collect sufficient RSS samples. It is therefore not applicable to highly dynamic networks, and its localization accuracy is lower than the RSS ranging based algorithms [14]. Considering the RSS of a same AP can vary a lot in two rooms, [15] proposes to utilize this characteristic to distinguish different rooms. [16] studies a Sybil attack detection method based on the RSS signature. A secure key generation method is designed in [3] based on the difference of RSS between two antennas, but it only works in a small range $(20 \mathrm{~cm})$.

The performance of RSS based applications accounts on the RSS properties such as stability and predicability. However, it has been shown that RSS are generally unstable and unpredictable in reality [4], [5]. [4] shows that it is impossible to obtain an accurate RSS value because of the multipath effect. [5] demonstrates the time-varying nature of RSS. In order to overcome these problem and to improve the performance of RSS based applications, several RSS processing methods have been proposed in [3], [17]-[19]. [18] proposes to use CSI (Channel State Information) from OFDM systems for improving the indoor localization accuracy. [19] tries to mine the phase information via exploiting the frequency diversity of the radio propagation paths. However, both of them are hardware dependent and thus limit their applications. [17] shows that the variability of RSS can be reduced by averaging the measured RSS from multiple antennas that are deployed at fixed locations. [3] propose to employ two antennas to reduce the variability of RSS and predict the RSS related outputs. As the path loss exponent still affect their outputs, the outputs' stability and predicability are still not satisfactory and result in the limitations on the applicability of the proposed works. Unlike these works, the proposed RSS-Ratio in this paper have eliminated all the unpredictable environmental parameters including the path loss exponent, and its stability and predicability have been justified via experiments, analysis, and simulations.

\section{CONCLUSIONS}

In this paper, we proposed a RSS processing method, named RSS-Ratio, to improve the performance of RSS-based wireless applications under both the Find and the Record methodologies. The proposed RSS-Ratio has been justified to be more stable and predictable than the original RSS via analysis, simulation, and both indoor and outdoor experiments, where the impacts of sender location, receiving antenna location, transmission power, frequency, and interference have been examined. In addition, as all the equations for converting RSS readings to $\mathrm{dBm}$ are linear, RSS-Ratio can also eliminate their differences, and may be identical cross platforms. We will further investigate it via experiments, where various antennas and platforms will be deployed at a same location in the future work.

\section{ACKNOWLEDGMENTS}

We would like to acknowledge and extend our heartfelt gratitude to the following persons who have made the completion of this paper possible: Our colleague, Dr. Parth Pathak, for his invaluable comments on preparing the paper. Carlo Vallati, visiting student from Dipartimento di Ingegneria dell'informazione, University of Pisa, for helping us set up the QuRiNet experiments. The research was partially supported by the US National Science Foundation under grants CNS1248380 , CNS-1265311.

\section{REFERENCES}

[1] J. geun Park, D. Curtis, S. Teller, and J. Ledlie, "Implications of device diversity for organic localization," in INFOCOM, 2011 Proceedings IEEE, april 2011, pp. $3182-3190$.

[2] K. Whitehouse, C. Karlof, and D. Culler, "A practical evaluation of radio signal strength for ranging-based localization," SIGMOBILE Mob. Comput. Commun. Rev., vol. 11, pp. 41-52, January 2007.

[3] L. Cai, K. Zeng, H. Chen, and P. Mohapatra, "Good neighbor: Adhoc pairing of nearby wireless devices by multiple antennas," in NDSS Symposium 2011, Feb. 2011.

[4] K. Wu, H. Tan, H. Ngan, Y. Liu, and L. Ni, "Chip error pattern analysis in ieee 802.15.4," Mobile Computing, IEEE Transactions on, vol. PP, no. 99 , p. 1, 2011.

[5] G. Zhou, T. He, S. Krishnamurthy, and J. A. Stankovic, "Impact of radio irregularity on wireless sensor networks," in Proceedings of the 2nd international conference on Mobile systems, applications, and services, ser. MobiSys '04. New York, NY, USA: ACM, 2004, pp. 125-138.

[6] T. S. Rappaport, Wireless Communications: Principles and Practice, 2nd ed. Prentice Hall, January 2002.

[7] J. Goldhirsh and W. J. Vogel, Handbook of Propagation Effects for Vehicular and Personal Mobile Satellite Systems -Overview of Experimental and Modeling Results. APL \& EERL, 1998.

[8] A. Goldsmith, Ed., Wireless Communications. Cambridge University Press, August 2005.

[9] R. University, "Warp: Wireless open access research platform." [Online]. Available: http://warp.rice.edu/trac/

[10] "The madwifi project." [Online]. Available: http://madwifi-project.org/

[11] D. Wu, D. Gupta, and P. Mohapatra, "Qurinet: A wide-area wireless mesh testbed for research and experimental evaluations," Ad Hoc Networks, vol. 9, no. 7, pp. 1221 - 1237, 2011.

[12] F. Fainelli, "The openwrt embedded development framework," in Proceedings of the Free and Open Source Software Developers European Meeting, 2008

[13] T. Clausen and P. Jacquet, "Rfc 3626-optimized link state routing protocol (olsr)," IETF RFC3626, 2003.

[14] B. Dil and P. Havinga, "On the calibration and performance of rss-based localization methods," in Internet of Things (IOT), 2010, 29 2010-dec. 12010 , pp. $1-8$.

[15] Y. L. Chenshu Wu, Zheng Yang and W. Xi, "Will: Wireless indoor localization without site survey," in INFOCOM, 2012 Proceedings IEEE, march 2012, pp. $64-72$.

[16] M. Demirbas and Y. Song, "An rssi-based scheme for sybil attack detection in wireless sensor networks," in Proceedings of the 2006 International Symposium on on World of Wireless, Mobile and Multimedia Networks, ser. WOWMOM '06. Washington, DC, USA: IEEE Computer Society, 2006, pp. 564-570.

[17] K. Kleisouris, Y. Chen, J. Yang, and R. Martin, "Empirical evaluation of wireless localization when using multiple antennas," Parallel and Distributed Systems, IEEE Transactions on, vol. 21, no. 11, pp. 1595 -1610 , nov. 2010.

[18] K. Wu, J. Xiao, Y. Yi, M. Gao, and L. Ni, "Fila: Fine-grained indoor localization," in INFOCOM, 2012 Proceedings IEEE, March 2012.

[19] D. Zhang, Y. Liu, X. Guo, M. Gao, and L. M. Ni, "On distinguishing the multiple radio paths in rss-based ranging," in INFOCOM, 2012 Proceedings IEEE, march 2012, pp. $2201-2209$. 